ISSN electrónico: 2445-1355

DOI: http://dx.doi.org/10.14201/fj2020515563

\title{
HÁBITOS DE ACTIVIDAD FÍSICA Y FACTORES RELACIONADOS EN ADOLESCENTES
}

\section{Habits of physical activity and related factors in teenagers}

Estela MARTÍN GÓMEZ; Ramona MATEOS CAMPOS

Área de Medicina Preventiva y Salud Pública. Departamento de Ciencias Biomédicas y del Diagnóstico. Facultad de Medicina. Universidad de Salamanca. Campus Miguel de Unamuno. C/ Alfonso X El Sabio, s/n, 37007 Salamanca

Correo-e: rmateos@usal.es

RESUMEN: La relación entre la práctica de actividad física y la alimentación en adolescentes permite establecer patrones de vida saludables que mejoran la salud en la edad adulta.

Este estudio describe los hábitos de actividad física de un grupo de adolescentes divididos por la edad, a los que se aplicó una encuesta de 19 preguntas para determinar intensidad, frecuencia y tipo de actividad física; evaluar los niveles de vida sedentaria, y valorar el patrón alimentario.

Existen diferencias significativas en la frecuencia e intensidad del ejercicio físico, los chicos realizan un ejercicio vigoroso-enérgico, mientras que las chicas realizan un ejercicio moderado.

Durante el tiempo libre, un pequeño grupo de chicos realizan deporte y las chicas dan un paseo, sin embargo, la mayoría prefiere usar su móvil.

Una significativa proporción de adolescentes no desayunan por la mañana, principalmente chicas. Existen diferencias en el patrón alimentario en el consumo de carne, fruta, galletas o bollos.

Aunque la mayoría de adolescentes practican actividad física, no son suficientemente activos y, con la edad, empeoran los hábitos, por lo que se debe concienciar de mantener una práctica diaria, disminuir las horas con el móvil y mejorar los hábitos alimentarios.

Palabras clave: actividad física; adolescentes; alimentos; tiempo; frecuencia. 
ABSTRACT: The relationship between the practice of physical activity and eating habits in adolescents allow to establish healthy life patterns that improve health in adulthood.

This study describes the physical activity habits of a group of adolescents divided by age, to whom a questionnaire of 19 questions was applied to determine the intensity, frequency and type of physical activity, evaluate the sedentary life levels, and assess the food pattern.

There are significant differences in the frequency and intensity of physical exercise, boys perform a vigorous-energetic exercise, while girls carry out moderate exercise.

During free time, a small group of boys perform sports and the girls take a walk, however, most prefer to use their mobile.

A significant proportion of adolescents do not have breakfast in the morning, mainly girls. There are differences in the eating pattern in the consumption of meat, fruit, cookies or buns.

Although most adolescents practice physical activity, they are not active enough and, with age, habits worsen, so they must be aware of maintaining a daily practice, reducing hours with their mobile phones and improving their eating habits.

Keywords: physical activity; teenagers; food; time; frequency.

\section{INTRODUCCIÓN}

La adolescencia es una etapa decisiva en la adquisición y consolidación de los estilos de vida. Los adolescentes muestran especial curiosidad y receptividad, y aún son relativamente inexpertos (Chillón Garzón et al., 2002).

Los patrones de actividad física en adolescentes tienen importantes implicaciones para la salud, ya que, con bajos niveles de práctica puede haber un inicio precoz de cardiopatías, de osteoporosis o de obesidad en la edad adulta (AbarcaSos et al., 2010).

Ligadas a la práctica de actividad física aparecen ciertas conductas sedentarias que ocupan las horas de ocio de los adolescentes, como son el uso del ordenador, del teléfono móvil, de los videojuegos y las horas delante de la pantalla del televisor (Portolés, González, 2015).

La práctica habitual de actividad física y una adecuada alimentación se presentan como hábitos fundamentales para la vida diaria de las personas, ya que ambas conductas permiten conservar o mejorar la calidad de vida relacionada con la salud (Pérez-López et al., 2015). 


\section{Objetivos}

\subsection{Objetivo general}

Conocer los hábitos de actividad física y factores relacionados en adolescentes.

\subsection{Objetivos especificos}

1. Analizar la práctica de actividad física teniendo en cuenta variables epidemiológicas de persona: edad y sexo.

2. Conocer la intensidad, frecuencia y tipo de actividad física que realizan los adolescentes.

3. Evaluar los niveles de vida sedentaria.

4. Valorar el patrón alimentario.

\section{Materiales y métodos}

1. Tipo de estudio: estudio epidemiológico descriptivo (Piédrola Gil, 2015).

2. Fuente de datos: se realizó un cuestionario de 19 preguntas (Anexo 1) en el que se incluyeron los siguientes apartados:

- Características epidemiológicas de persona.

- Práctica de actividad física.

- Hábitos sedentarios.

- Hábitos alimenticios.

3. Población de estudio: en total se incluyen 118 alumnos del IES La Sisla (Sonseca), dividiéndose en dos grupos:

- Grupo 1: formado por 76 alumnos de $10^{\circ}$ y $2 .^{\circ}$ ESO.

- Grupo 2: formado por 42 alumnos de $4 .^{\circ} \mathrm{ESO}$ y $1 .^{\circ}$ Bachillerato.

\section{Variables de estudio:}

- Variables epidemiológicas de persona:

o Sexo.

o Edad.

- Variables dependientes:

o Práctica de actividad física: intensidad, tiempo y frecuencia.

o Actividades sedentarias: estudio, desplazamiento y ocio.

o Hábitos alimentarios: número de comidas al día, desayuno e ingesta de distintos tipos de alimentos. 
5. Se realizó un estudio piloto para la elaboración del cuestionario definitivo.

6. Aplicación del cuestionario: se llevó a cabo durante el mes de mayo del 2018 en una visita a las distintas aulas acompañada de los tutores de las clases.

7. Análisis de los resultados: se calcularon las distintas frecuencias y porcentajes para elaborar las tablas y figuras mediante una base de datos en Microsoft Excel. La posible relación entre variables se valora mediante el estadístico «chi cuadrado» y el nivel $\rho$ de significación.

\section{Resultados}

\subsection{Características de la muestra}

El total de la muestra fueron 118 adolescentes, donde el 52,5\% son hombres y el $47,5 \%$ mujeres. El 64,4\% de los estudiantes encuestados tienen entre 12-14 años, de los cuales el $69,4 \%$ son chicos y el $58,9 \%$ chicas. A su vez, el $35,6 \%$ tienen entre 15-18 años, con el 30,6\% de hombres frente al 41,1\% de mujeres

\subsection{Valoración de la práctica de actividad física según el sexo}

De todos los estudiantes, el 10,2\% no practica actividad física, siendo mayoritario el grupo de mujeres (16,1\%). Respecto a la frecuencia, el $24,6 \%$ practica actividad física cada día, destacando el grupo masculino (33,9\%); el 53,4\% la realizan 2-3 días a la semana, y el 11,9\% solo el fin de semana (Figura 1) (diferencias

Figura 1: Frecuencia de la práctica de actividad física según el sexo (\%)

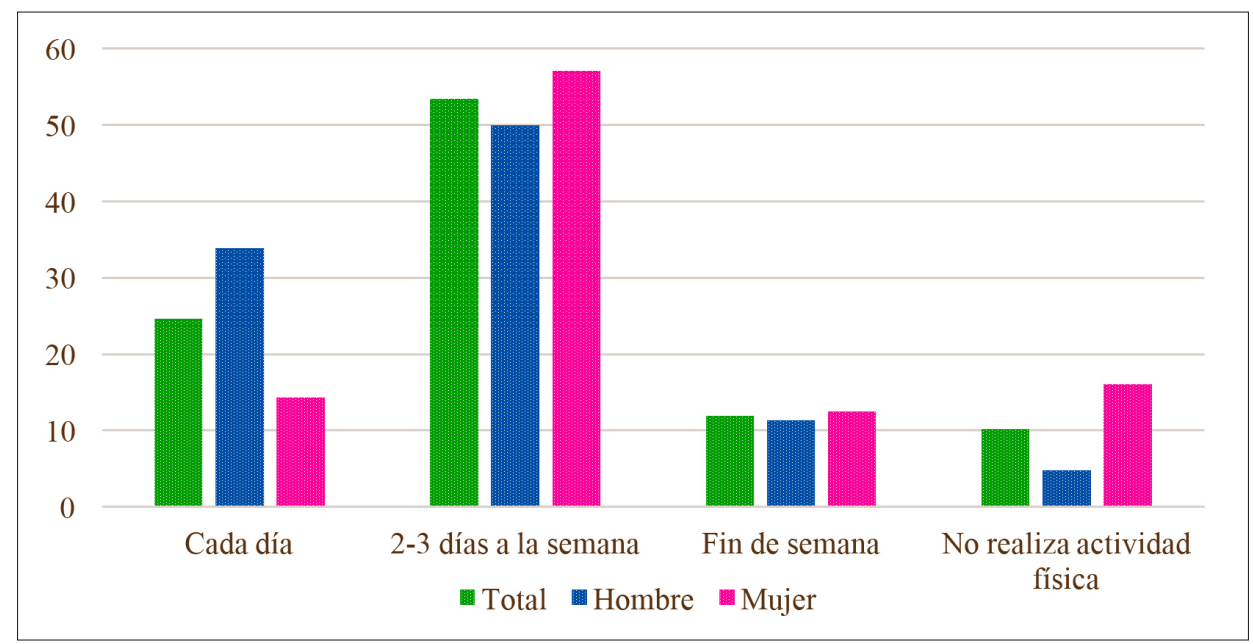


significativas $p=0,036$ ), resultados que también señalan otros estudios (BeltránCarillo et al., 2012; Cano Garcinuño et al., 2011).

El tiempo que dedican a la práctica de ejercicio físico, el 14,9\% de las adolescentes está alrededor de 30 minutos y el $27,7 \%$ entre los 30 minutos y 1 hora. Por otro lado, los que más tiempo dedican a la actividad física son los adolescentes, ya que el 18,6\% practican 1 hora y el 57,6\% más de 1 hora.

En cuanto a la intensidad del ejercicio, las mujeres realizan ejercicio moderado $(57,4 \%)$, mientras que los hombres un ejercicio vigoroso-enérgico $(52,5 \%)$.

\subsection{Valoración de la práctica de actividad física según la edad}

Los adolescentes entre 15-18 años son los que mayoritariamente no realizan actividad física (16,7\%). Respecto a la frecuencia, tanto el grupo entre 12-14 años como el grupo de mayor edad (15-18 años) realizan actividad física, mayoritariamente, entre 2 y 3 días a la semana.

En cuanto al tiempo que realizan actividad física, los adolescentes entre 12-14 años dedican más de 1 hora (45,1\%), seguido de aquellos que dedican 1 hora (22,5\%). Igual ocurre en los adolescentes de 15-18 años, pues el 60\% practica actividad física más de 1 hora seguido del $25,7 \%$, que dedica entre 30 minutos y 1 hora.

Respecto a la intensidad, los chicos entre 12-14 años realizan un ejercicio moderado $(50,7 \%)$, seguido de los que lo realizan vigoroso-enérgico $(39,4 \%)$. Entre los 15-18 años, practican un ejercicio moderado el 48,6\% y ejercicio vigoroso-enérgico el $42,9 \%$.

En el tipo de ejercicio, la mayoría de los estudiantes practican deportes de equipo (41,5\%): fútbol, baloncesto o balonmano. También destacan otras actividades como correr $(18,9 \%)$, natación $(14,2 \%)$ y baile $(13,2 \%)$.

$\mathrm{Al}$ estudiar el motivo por el que practican actividad física, la mayoría lo hace por diversión (72,6\%), más los chicos $(83,1 \%)$ que las chicas $(59,6 \%)$. Un pequeño porcentaje de mujeres practican actividad física para eliminar el estrés $(19,1 \%)$ o para adelgazar $(17,0 \%)$.

La práctica de actividad física debe combinarse con horas de estudio o los deberes. Al 21,4\% de los estudiantes entre 15-18 años, el estudio les impide frecuentemente practicar actividad física mientras que al $42,9 \%$ solo en ocasiones. Al 36,8\% de los adolescentes entre 12-14 años sólo en ocasiones el estudio les impide realizar actividad física mientras que al 30,3\% nunca se lo impide. 


\subsection{Valoración de los hábitos sedentarios}

Dentro del modo de desplazamiento usado por los estudiantes para ir a clase, destaca el coche $(35,6 \%)$, seguido del uso de la bicicleta $(29,7 \%)$, ir andando $(20,3 \%)$ o en autobús $(14,4 \%)$. Existen diferencias significativas, $p=0,05$, entre hombres y mujeres.

Los chicos entre 12-14 años prefieren ir a clase en bicicleta (35,5\%), seguido de los que prefieren ir en coche (31,6\%). Entre los 15-18 años, prefieren ir en coche $(42,9 \%)$ y, en menor porcentaje, van andando $(21,4 \%)$.

En general, el 51,7\% de los estudiantes dedican 1-2 horas a estudiar o a leer un libro cada día, mientras el 23,8\% entre los 15-18 años dedica 3-4 horas.

El 48,4\% de los chicos dedican de 1 a 2 horas a hacer los deberes al igual que el $55,4 \%$ de las chicas. Sin embargo, mientras el $38,7 \%$ de ellos dedican menos de 1 hora, el 26,8\% de ellas, entre 3-4 horas.

$\mathrm{Al}$ analizar las actividades en el tiempo libre, el 39,8\% de los adolescentes mira el móvil, la tablet o el ordenador, el $41,9 \%$ de los chicos prefiere la práctica de un deporte o ejercicio, y el 23,2\% de las chicas prefiere dar un paseo. En menor medida, un 9,7\% de ellos aprovecha para ver la tele y el 14,3\% de ellas leen un libro.

Respecto a la edad, el 39,5\% de los chicos entre 12-14 años prefieren estar con el móvil, ordenador o tablet en su tiempo libre, seguido del $34,2 \%$ que prefiere practicar deporte o ejercicio físico. El 40,5\% de los adolescentes entre 15-18 años dedican su tiempo libre a mirar su móvil, ordenador o tablet y, en menor medida, el 26,2\% prefieren dar un paseo.

El uso del móvil, ordenador o ver la televisión son los principales hábitos sedentarios en los adolescentes. El 45,8\% pasan entre 1-2 horas con el móvil, seguido de un $22,9 \%$ que lo utiliza entre 3-4 horas.

Los adolescentes entre 12-14 años pasan entre 1-2 horas con el móvil, ordenador o viendo la televisión (53,9\%), y también el 31,0\% de los chicos entre 15-18 años. Diferencias significativas $p=0,0365$ (Figura 2).

\subsection{Valoración de los hábitos alimentarios}

El 57,6\% de los encuestados realizan 3-4 comidas al día, aunque cabe destacar que el $46,4 \%$ de las mujeres realizan las 5 comidas diarias mientras que el 6,5\% de los hombres realizan menos de 3 comidas al día.

Considerando la importancia del desayuno, analizamos los alimentos que suelen ingerir durante el mismo. El 11,9\% de los adolescentes reconocen que no desayunan por las mañanas, el $8,1 \%$ de los chicos y el $16,1 \%$ de las chicas; tampoco desayunan el 10,5\% de chicos entre 12-14 y, el 14,3\% de los de 15-18 años. 
Los adolescentes que desayunan, el 50\% toman leche, yogur o algún derivado lácteo, el 43,2\% consumen galletas, cereales o productos de bollería, y, en menor medida, el 13,6\% prefiere fruta y el 13,6\% zumos.

El 41,5\% de los adolescentes ingieren más de un alimento durante el desayuno, lo más frecuente, leche y galletas $(59,2 \%)$. En menor medida, también combinan leche, galletas, zumos y fruta $(8,2 \%)$; leche, galletas y zumos $(6,1 \%)$, o galletas y zumos $(4,1 \%)$, resultados similares a otros estudios (Agencia Española de Consumo, 2016).

Figura 2: Horas ordenador, televisión o móvil en función de la edad (\%)

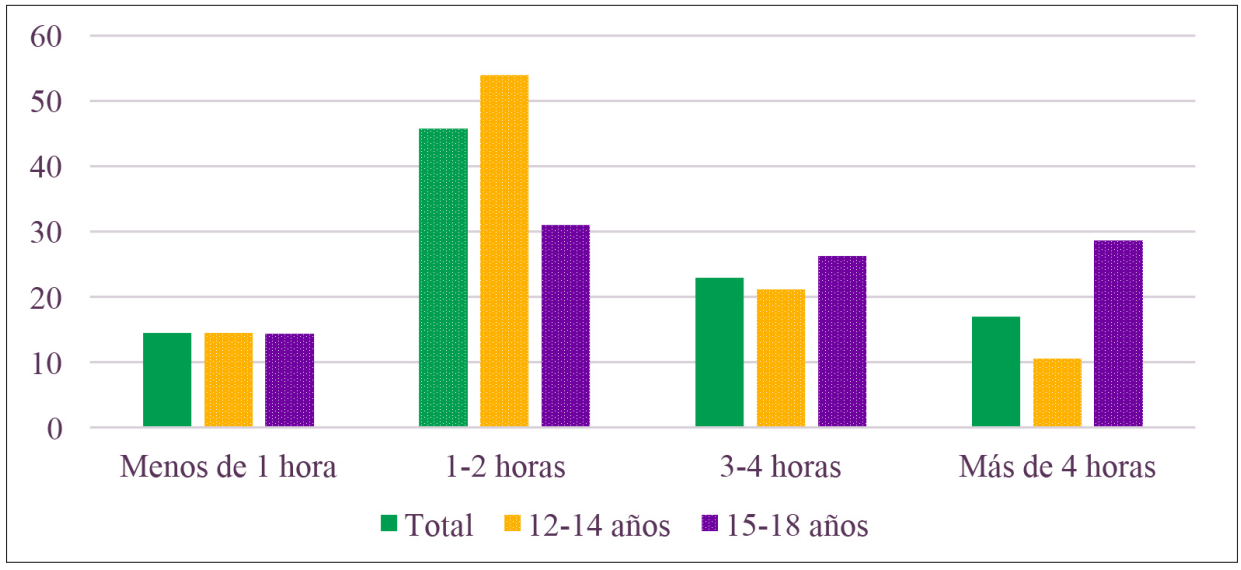

Figura 3: Frecuencia de consumo de los alimentos por semana (\%)

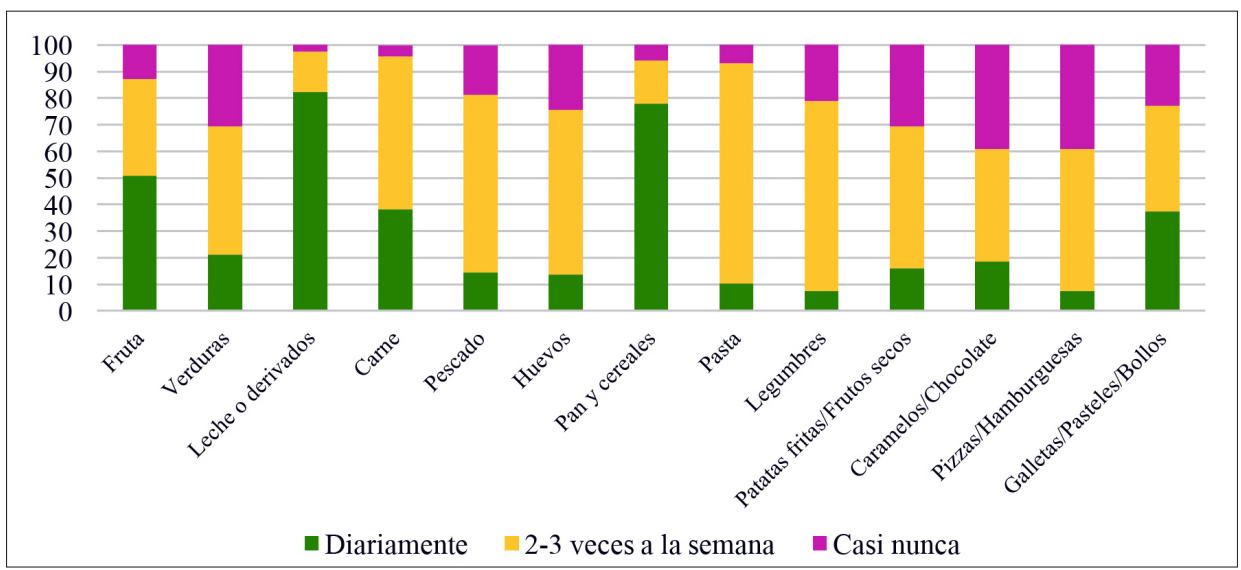

Ediciones Universidad de Salamanca / @®@ 
Respecto a la frecuencia del consumo de diferentes tipos de alimentos, el $82,2 \%$ de los chicos consume a diario leche y el $78 \%$ pan. Entre los alimentos que consumen de $2-3$ veces a la semana se encuentran: pescado $(66,9 \%)$, huevos $(61,9 \%)$, legumbres $(71,2 \%)$, pasta $(83 \%)$ y patatas fritas o frutos secos $(53,4 \%)$ (Figura 3).

Así mismo, también se analizó la frecuencia del consumo de diferentes tipos de alimentos tanto en hombres como en mujeres, destacando que el $23,2 \%$ de las mujeres casi nunca come fruta frente al $59,7 \%$ de los hombres que la consume a diario. El 56,8\% de los chicos toma carne a diario mientras que el $64,3 \%$ de las chicas 2-3 veces a la semana. El $21 \%$ de los hombres toman patatas fritas y frutos secos a diario y más de la mitad (53,2\%) consume galletas, pasteles o bollos 2-3 veces a la semana, mientras que el $33,9 \%$ de las mujeres casi no lo consume.

\section{Conclusiones}

1. La mayoría de adolescentes realizan actividad física 2-3 días a la semana y los que no realizan actividad física son, en su mayoría, chicas.

2. Los adolescentes participan en deportes de equipo, aunque los chicos realizan un ejercicio vigoroso-enérgico durante más de 1 hora, mientras que las chicas realizan un ejercicio moderado entre 30 minutos y 1 hora.

3. No existen diferencias en la práctica de actividad física en los distintos grupos de edad, aunque disminuye con la edad.

4. Los adolescentes suelen ir al instituto en coche, principalmente las chicas y los chicos de mayor edad, mientras que los más jóvenes prefieren ir en bicicleta.

5. Durante el tiempo libre, los adolescentes prefieren usar su móvil, ordenador o ver la televisión 1-2 horas, actividades sedentarias, aunque un pequeño grupo de chicos prefieren realizar deporte y las chicas dar un paseo.

6. La ingesta ocasional de caramelos, chocolate, pizzas o hamburguesas, así como el consumo de carne 2-3 veces a la semana o la ingesta a diario de leche y pan, son buenos hábitos alimentarios, pero una significativa proporción de adolescentes no desayunan, principalmente chicas.

7. Aunque la mayoría de adolescentes practican actividad física, no son suficientemente activos y, con la edad empeoran los hábitos, por lo que se debe concienciar de mantener una práctica diaria, disminuir las horas con el móvil y mejorar los hábitos alimentarios. 


\section{Bibliografía}

Abarca-Sos A, Zaragoza Casterad J, Generelo Lanaspa E, Julián Clemente JA. Comportamientos sedentarios y patrones de actividad física en adolescentes. Rev Int Med Cienc Act Fís Deporte. 2010; 10(39):410-427.

Agencia Española de Consumo, Seguridad Alimentaria y Nutrición. Estudio ALADINO 2015: Estudio de Vigilancia del Crecimiento, Alimentación, Actividad Física, Desarrollo Infantil y Obesidad en España 2015. Madrid: Ministerio de Sanidad, Servicios Sociales e Igualdad; 2016 [actualizado en 2015; citado 2 diciembre 2018]. Disponible en: http://www.aecosan.msssi.gob.es/AECOSAN/docs/documentos/nutricion/ observatorio/Estudio_ALADINO_2015.pdf.

Beltrán-Carrillo VJ, Devís-Devís J, Peiró-Velert C. Actividad física y sedentarismo en adolescentes de la Comunidad Valenciana. Rev Int Med Cienc Act Fís Deporte. 2012; 12(45):122-137.

Cano Garcinuño A, Pérez García I, Casares Alonso I, Alberola López S. Determinantes del nivel de actividad física en escolares y adolescentes: estudio OPACA. An Pediatr. 2011; 74(1):15-24.

Chillón Garzón P, Delgado Fernández M, Tercedor Sánchez P, González Gross M. Actividad físico-deportiva en escolares adolescentes. Retos. Nuevas Tendencias en Educación Física, Deporte y Recreación. 2002; (3):5-12.

Pérez-López IJ, Tercedor Sánchez P, Delgado-Fernández M. Efectos de los programas escolares de promoción de actividad física y alimentación en adolescentes españoles: revisión sistemática. Nutr Hosp. 2015; 32(2):534-544.

Piédrola Gil. Medicina Preventiva y Salud Pública. 12.a ed. Barcelona: Elsevier; 2015.

Portolés A, González J. Rendimiento académico y correspondencias con indicadores de salud física y psicológica. Sportis Scientific Technical Journal. 2015; 1(2):164-181.

\section{Agradecimientos}

Por su colaboración para realizar las encuestas en sus aulas al IES La Sisla (Sonseca) y a todos los adolescentes que han participado. 